\section{Viral assembly inhibitors on the horizon}

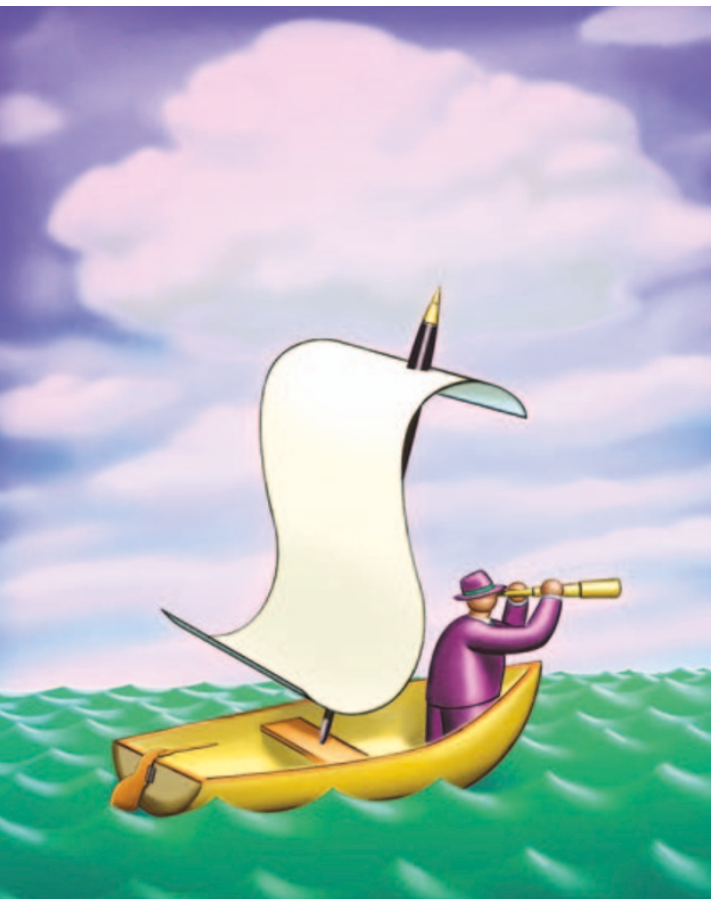

\section{ANTICANCER DRUGS}

\section{Double strike}

In an ideal world you could take the best parts of several different anticancer drugs and turn them into a more effective drug that is almost invincible against resistance. Researchers at the Massachusetts Institute of Technology have taken a step towards this goal by linking the DNAbinding activity of one class of drugs to the transcription-modulating activity of another class of molecule. The result, described in Chemistry and Biology, is a compound called $11 \beta$-dichloro that has anticancer activity against prostate cancer cells in vitro and in vivo.

The first modern anticancer drugs were alkylating agents that cause cell death by introducing adducts into DNA and disrupting cell division, and continue to be the mainstay of current chemotherapy regimens. These early therapies were toxic to patients, so attention has turned recently to the development of more sophisticated drugs targeting single proteins within signalling pathways. However, the development of resistance and the risk of drug interactions
Current therapy for human immunodeficiency virus (HIV)-infected patients includes drugs that block the viral enzymes reverse transcriptase and protease. In addition, some combination therapies include a relatively new viral-entry inhibitor. Now, scientists have identified a molecule that interferes with the formation of HIV particles in vitro, according Biology. These findings could lead to the development of a new class of HIV drugs that slow or prevent the progression of HIV in patients.

More than 40 million people globally are currently infected with HIV, the causative agent of AIDS, and approximately 5 million people become infected each year. Although combination therapy has been highly successful, the emergence of HIV strains resistant to more than one of the drugs in these regimes has made the identification of alternative targets a high priority.

HIV-1 is released from infected cells as immature, non-infectious particles with a protein shell composed of Gag proteins. Formation

associated with taking several agents together suggests that targeting multiple pathways using a single multifunctional compound might be a better approach.

Having previously developed compounds that generate DNA adducts that sequester the oestrogen receptor with high affinity, John Essigmann, Robert Croy and colleagues speculated that it should be possible to custom-design analogues that produce DNA adducts while also antagonizing the androgen receptor (AR), a nuclear receptor required for prostate cancer growth. The authors proposed that a bifunctional molecule consisting of a DNA damaging agent tethered to an AR ligand would sequester the $A R$ at the site of the DNA adduct, thereby protecting the AR signalling - resulting in a two-pronged attack on prostate cancer cells.

The bifunctional agent, $11 \beta$-dichloro, consisted of an anti-androgenic compound, $17 \beta$-hydroxy-estra- $\Delta^{4(5), 9(10)}$-3-one, and a DNA alkylator molecule, $N, N$-bis-2-chloroethyl aniline. As hoped, $11 \beta$-dichloro displayed potent cytotoxic effects against $\mathrm{LNCaP}$ prostate tumour cells caused by a rapid induction of apoptosis and upregulation of cell-cycle checkpoint proteins. These key biochemical sentinels of cytotoxic activity were not triggered by the distinct chemical to two papers in Nature Structural \& Molecular DNA adducts from repair while antagonizing of the infectious particles requires the cleavage of Gag into a number of smaller proteins, including matrix, capsid (CA) and nucleocapsid. The final processing step, which occurs inside the virion, releases CA from an adjacent spacer. Processed CA molecules then interact to form the conical capsid of the mature, infectious virus.

In the first paper, Hans-Georg Kräusslich and colleagues report using phage-display screening to identify peptides within the CA molecule that bind tightly and specifically to CA and which might thereby affect its interactions with other CA proteins. Three selected peptides were synthesized and their ability to compete with peptide-presenting phages for binding to CA was tested. One peptide, CAI, efficiently inhibited both immature and mature particle assembly in vitro with a binding constant in the micromolar range. NMR analysis revealed the binding site of the peptide.

In the second paper, Felix Rey and colleagues report the $1.7 \AA$-resolution crystal structure of the carboxy-terminal domain of CA (C-CA) in complex with CAI. The peptide inserts as an additional $\alpha$-helix into a conserved hydrophobic groove at C-CA, resulting in the formation of a compact five-helix complex. This interaction alters the CA dimer interface, presumably leading to non-functional dimers

entities from which the drugs were made - that is, the DNA-damaging agent or the receptor ligand. Both elements of the bifunctional $11 \beta$-dichloro were required for this response.

In a tumour xenograft model in which LNCaP cells were grafted into nude mice, a striking $90 \%$ inhibition of tumour growth was observed after a 7 -week treatment with $11 \beta$-dichloro. Further studies with larger, more established tumours are now being carried out by these authors to study whether the cytotoxic effects observed in vitro can be translated to a reduction in tumour size. Other potential refinements include modification of the AR ligand portion of the compound to increase its affinity for other $A R$ variants that are common in prostate malignancies. Although there have been examples of the use of tethering to target drugs to tumours or other tissues, this is a fine example of how the properties of two drugs can be combined using a chemically stable linkage to make a smarter, more effective therapeutic.

Joanna Owens

\section{(6) References and links} ORIGINAL RESEARCH PAPER

Marquis, J. C. et al. Disruption of gene expression and induction of apoptosis in prostate cancer cells by a DNA damaging agent tethered to an androgen receptor ligand. Chem. Biol. 12, 779-787 (2005) 
that interfere with formation of viral capsids. As such, the structure reveals the details of an allosteric site in CA that can be targeted by small-molecule inhibitors.

These studies identify the first inhibitor directed against the formation of immature as well as mature HIV-1 particles, and could be developed for antiviral treatment. Unfortunately, CAI itself cannot be used therapeutically; as with many peptides, the half-life is short and the bioavailability is poor. However, the peptide does represent a suitable starting point for drug development.

Melanie Brazil

6) References and links ORIGINAL RESEARCH PAPERS Sticht, J. et al. A peptide inhibitor of $\mathrm{HIV}-1$ assembly in vitro. Nature Struct. Mol. Biol. 12, 671-677 (2005) | Ternois, F. et al. The HIV-1 capsid protein C-terminal domain in complex with a virus assembly inhibitor. Nature Struct. Mol. Biol. 12, 678-682 (2005) FURTHER READING Pommier, Y., Johnson, A. A. \& Marchand, C. Integrase inhibitors to treat HIV/ AIDS. Nature Rev. Drug Discov. 4, 236-248 (2005) Matthews, T. et al. Enfuvirtide: the first therapy to inhibit the entry of HIV-1 into host CD4 lymphocytes. Nature Rev. Drug Discov. 3, 215-225 (2004) I Stone, A. Microbicides: a new approach to preventing HIV and other sexually transmitted infections. Nature Rev. Drug Discov. 1, 977-985 (2002)

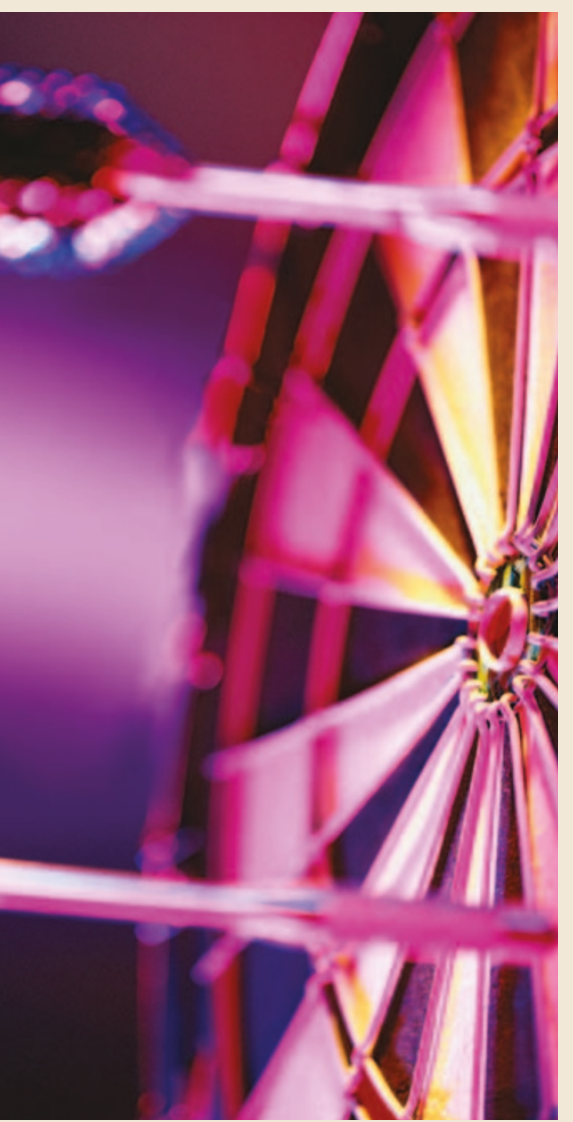

\section{Knocked down for the count}

After the initial excitement surrounding the use of RNA interference (RNAi) as a potential therapeutic modality, there have been substantial hurdles to developing RNAi-based drug candidates. Because unmodified short interfering RNAs (siRNAs) are highly unstable in vivo, the first hurdle was the need to create therapeutically viable siRNAs with improved stability, potency and duration of effect in humans. At least one chemically modified siRNA is in human clinical trials, which demonstrates the success of industry efforts in producing stable, effective therapeutic agents. There remains, however, another hurdle to the widespread application of siRNA to human disease: systemic delivery, and particularly the need to reduce the large doses of siRNAs currently required to achieve prolonged mRNA knockdown. But progress is finally being made on this front. In a recent issue of Nature Biotechnology, David Morrissey and colleagues describe how a stable nucleic-acid-lipid particle (SNALP) containing chemically stabilized siRNA causes persistent knockdown of hepatitis $B$ virus (HBV) replication genes in mice.

Morrissey and colleagues had previously reported that stabilizing siRNAs by substituting all $2^{\prime}-\mathrm{OH}$ residues on the $\mathrm{RNA}$ with $2^{\prime} \mathrm{F}, 2^{\prime} \mathrm{O}-\mathrm{Me}$ or $2^{\prime} \mathrm{H}$ residues dramatically improves the in vivo stability of siRNA. However, the dosing regimen used to demonstrate this in a mouse model of $\mathrm{HBV}$ replication was not therapeutically viable because it required administration of large amounts of siRNA three times daily. By encapsulating the stabilized siRNA in a specialized liposome, the authors were able to use a lower dose while improving the potency and duration of siRNA activity.

SNALPs consist of a lipid bilayer comprising cationic and fusogenic lipids, which enable cellular uptake and endosomal release of the siRNA, coated with a polyethylene glycol (PEG)-lipid conjugate that shields and stabilizes the particle, thereby preventing rapid clearance. When intravenously injected, the PEG-lipid conjugate dissociates from the SNALP particle, which can then go on to transfect cells in vivo.

Initial biodistribution and pharmacokinetic studies showed that substantial levels of SNALP accumulated in the liver and spleen of injected mice and that the siRNA-SNALP had a significantly longer half-life in the liver than the stabilized siRNA particle alone. In mice

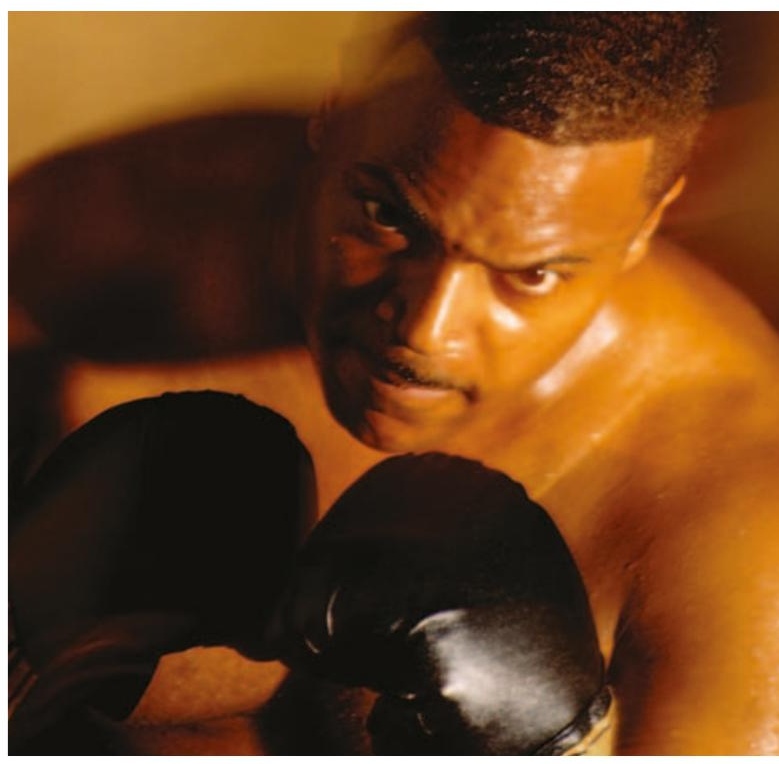

undergoing chronic treatment with SNALP, these pharmacokinetics were maintained throughout a 5-week treatment protocol.

The authors then tested the efficacy of siRNASNALP in a mouse model of HBV replication. Substantial dose-dependent reductions in serum HBV DNA levels were observed 7 days after administration of anti-HBV siRNA-SNALP and, importantly, similar reductions in $\mathrm{HBV}$ replication were maintained over a 6-week course of treatment. A more potent and persistent siRNA-mediated gene-silencing effect was achieved using lower and fewer doses than has been reported previously in the literature.

One well-documented issue with RNAi-based therapeutics is the induction of type 1 interferon inflammatory cytokine immune responses in mammalian cells. Morrissey and colleagues tested the potential for chemically modified siRNA-SNALPs to stimulate such a response, and found that, unlike the unmodified siRNA alone, the chemically modified siRNAs used in the SNALP formulation did not induce interferon- $\alpha$ and inflammatory cytokines in the serum of injected mice.

The authors speculate that the SNALP formulation could minimize the chances of developing oligonucleotide-related toxicities associated with antisense therapies, which have generally been related to dose. Their results are an important step towards making RNAi-based therapeutics a reality.

Joanna Owens

(9) References and links ORIGINAL RESEARCH PAPER

Morrissey, D. V. et al. Potent and persistent in vivo activity of chemically modified siRNAs. Nature Biotech. 23, 1002-1007 (2005) FURTHER READING Morrissey, D. V. et al. Activity of a stabilized short interfering RNA in a mouse model of hepatitis B virus replication. Hepatology 41, 1349-1356 (2005) | Dorsett, Y. \& Tuschl, T. siRNAs: applications in functional genomics and potential as therapeutics. Nature Rev. Drug Discov. 3, 318-329 (2004) 